\title{
Interpretation of the non-locality problem in quantum mechanics through the consciousness model
}

\author{
Dhananjay Pal \\ Email address: \\ dhananjay.pal123@gmail.com,paldhananjay46@yahoo.com
}

Pharmacy College, Bengal School of Technology, Sugandha-Delhi Road, Chuchura, Dist.-Hooghly, West Bengal, INDIA

\section{To cite this article:}

Dhananjay Pal. Interpretation of the Non-Locality Problem in Quantum Mechanics through the Consciousness Model. American Journal of Physics and Applications. Vol. 2, No. 1, 2014, pp. 1-5. doi: 10.11648/j.ajpa.20140201.11

\begin{abstract}
As per Penrose, consciousness is a part of the universe. Scientists have arrived at a simple but decisive conclusion that consciousness is very much a part of the universe, like other objects. Our consciousness model involving thought-carrying particle (TCP), thought retaining particle (TRP) and thought force $\left(\mathrm{T}_{\mathrm{F}}\right)$ signifies the existence of universal consciousness that exists along with the universe. This universal consciousness is a functional state of the universal mind. This universal mind (UM) is evolved at the Big Bang from void. The UM is constituted by these TCP and TRP in the inherent presence of thought force $\left(\mathrm{T}_{\mathrm{F}}\right)$. Thought force $\left(\mathrm{T}_{\mathrm{F}}\right)$ is an expression of universal consciousness. A single field emerged at the origin of the universe, already containing within itself the blueprint of the physical universe. The primordial single field triggered the onset of the universe. Most physicists believe that a single super-force dominated the first instants of creation. The Thought force $\left(\mathrm{T}_{\mathrm{F}}\right)$ being the primordial quantum field functions as the original super-force. $\mathrm{T}_{\mathrm{F}}$ being the original super-force functions as the origin of all the fundamental fields. TCP is the carrier of thought force $\left(\mathrm{T}_{\mathrm{F}}\right)$ that, in turn, appears to be the origin of all the fields. The quantized energy $\left(\varepsilon_{T}\right)$ of TCP is responsible to cause the universal consciousness as well as the cosmic microwave background radiation temperature. The individual consciousness owes its origin to the universal consciousness created by the same $\varepsilon_{T}$. The same $\varepsilon_{T}$ is the energy responsible for generating thought force $\left(\mathrm{T}_{\mathrm{F}}\right) . \mathrm{T}_{\mathrm{F}}$ being an expression of the universal consciousness is applicable to any inanimate object as well as to any biological system (having thinking ability). The $\mathrm{T}_{\mathrm{F}}$ exerts its functions both in vitro and in vivo. The universal consciousness exists throughout the universe in the form of universe wide web $(u w w)$ covering the universe as a whole with all its parameters (including void) and inhabitants (with or without consciousness). Everything in this universe is interlinked and intertwined through the existence of the quantum mechanical activities of these TCP in the inherent presence of TRP. Non-locality problem in quantum mechanics can be interpreted through the quantum mechanical activities of these TCP and TRP in the presence of thought force $\left(\mathrm{T}_{\mathrm{F}}\right)$ in vitro and thought force $\left(\mathrm{T}_{\mathrm{F}}\right)$ in vivo.
\end{abstract}

Keywords: Cosmic Microwave Background Radiation (CMBR), Universal Mind (UM), Thought Force $\left(\mathrm{T}_{\mathrm{F}}\right)$, Though-Carrying Particle (TCP), Thought Retaining Particle (TRP), Quantized Energy $\left(\varepsilon_{T}\right)$ of TCP

\section{Introduction}

In contrast to the usual linear sequence of matter, body, life, brain, mind, consciousness, here the proposed cyclic sequence is first universal consciousness (a functional state of the universal mind), and then matter, body, life, brain, and regeneration of mind and consciousness. The evolution of life with mind and consciousness is possible purely due to the inherent existence of universal consciousness which exists along with the universe. The human nervous system is evolved to provide an appropriate material structure to individualize the universal consciousness, a characteristic of reality, pervading all manifestations.

It is most relevant and important to indicate the names of various eminent physicists like Erwin Schrödinger, Eugene Wigner, Brian Josephson, John Wheeler, Roger Penrose, Henry P Stapp, Freeman J. Dyson, Paul Davies, David Bohm, Basil Hiley, Fritjof Capra, Fred Alan Wolf and Amit Goswami who have addressed the inclusion of consciousness in their work. Consciousness is to be taken into account.

As per Penrose [1], consciousness is a part of the universe. Van De Bogart [2] explained, "Since consciousness is a part of the universe it then follows that all consciousness, and the universe, are of the same matrix of energy fields".

Consciousness model of Pal et al [3-5] involving TCP, TRP and thought force $\left(\mathrm{T}_{\mathrm{F}}\right)$ signifies the existence of 
universal consciousness that exists along with the universe. Pal et al [5] showed that the functional state of Universal Mind (UM) is the universal consciousness that exists along with the universe. Pal et al [5] explained that the UM is evolved at the Big Bang from the eternal Void (see SchemeI). This Void, in turn, is the source of infinite energy. And this UM is a finer matter. The individual mind being a constituent of the UM is also a finer matter. The constituents of the UM and individual mind are the same. The ultimate constituents of matter and mind are the same as both mind and matter are aspects of one fundamental reality, which is called UM. The brain is the mediating link or interface between the individual mind and body.

Pal et al [5] explained that the constituents of the UM are the ultimate constituents of matter itself as everything in this universe is a manifestation of this UM. Pal et al [5] expressed that the UM is constituted by these TCP and TRP in the inherent presence of thought force $\left(\mathrm{T}_{\mathrm{F}}\right)$. Pal et al $[3,5]$ further explained that the ultimate constituents of matter and mind are these TCP and TRP in the inherent presence of thought force $\left(\mathrm{T}_{\mathrm{F}}\right)$ in vitro and thought force $\left(\mathrm{T}_{\mathrm{F}}\right)$ in vivo [See Scheme-I].

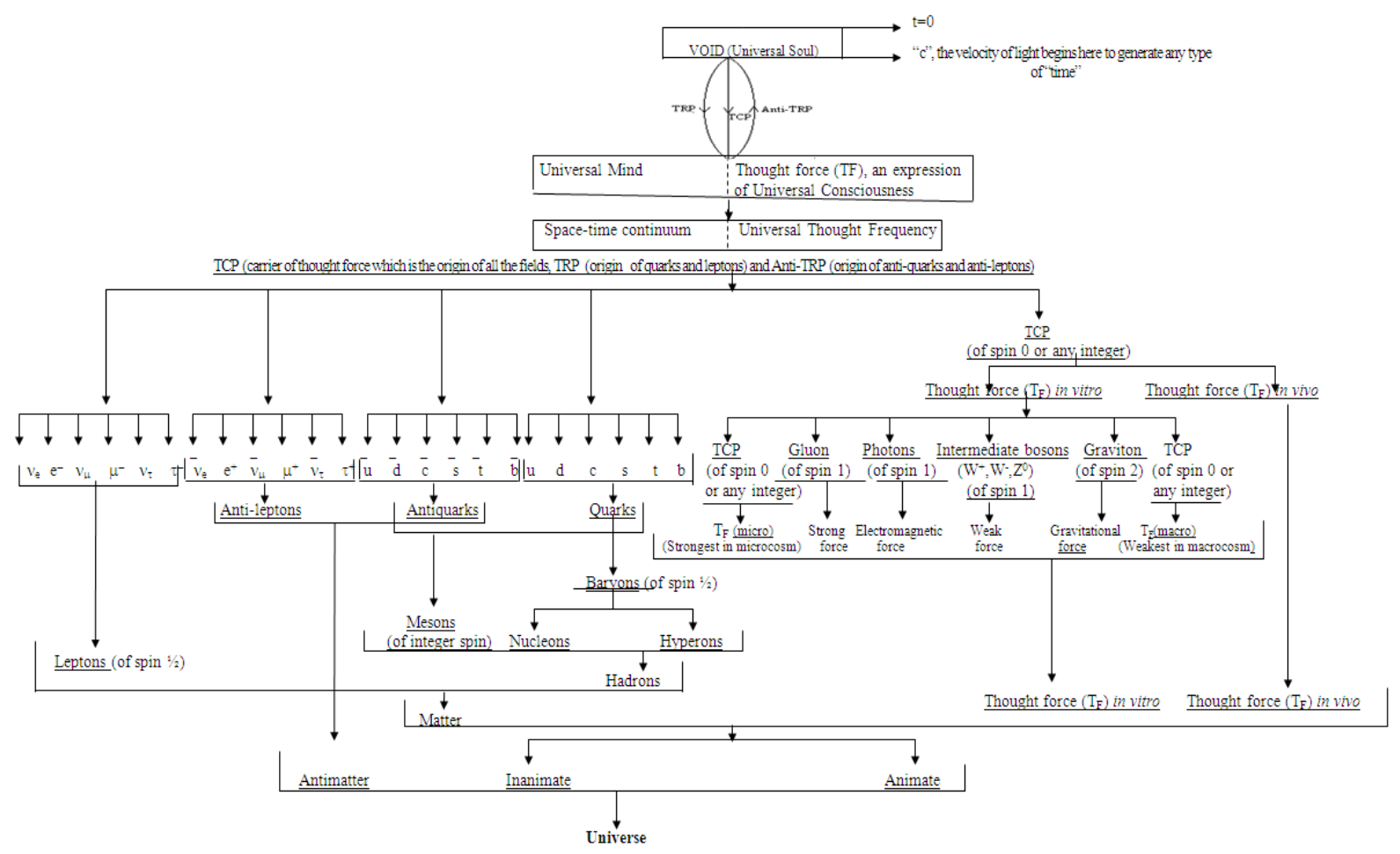

Scheme-I: Schematic presentation showing the evolution of the universe from the Void incorporating the postulated TCP (Thought-carrying particle), TRP (Thought-retaining particle) and Thought Force $\left(T_{F}\right)$. Laws of absolute conservation of mass, charge (and color) are to be maintained in order to develop a general theory for the unification of physics which would be freely applicable to the more general situations involving both the non-living system and living system having consciousness. Here TCP is the carrier of thought force ( $T_{F}$ ) that is the origin of all the fields. TRP is the origin of all the matter particles. It is to be noted that these TCP and TRP function like wavicle: wave-particle duality. Here thought force (TF) is an expression of the universal consciousness

\section{Evolution of Universe}

\subsection{General View for the Evolution of the Universe}

Big Bang $\rightarrow$ Universe $\rightarrow$ [Fields + Particles \& Antiparticles + STC $] \rightarrow[$ Matter and Fields + STC $] \rightarrow$ Matter and Fields + STC + Life as well as consciousness.

If the universe exists along with the universal consciousness that, in turn, is a functional state of the universal mind (UM) and if this UM is evolved at the Big Bang from the eternal void, then

\subsection{Our View for the Evolution of the Universe (See Scheme-I)}

VOID $\rightarrow$ [Big Bang $] \rightarrow[$ TCP, TRP \& Anti-TRP in the inherent presence of thought force $\left.\left(\mathrm{T}_{\mathrm{F}}\right)+\mathrm{STC}\right] \rightarrow$
[Universal Mind with universal consciousness + TCP \& TRP in the inherent presence of thought force $\left(\mathrm{T}_{\mathrm{F}}\right)+\mathrm{STC}+$ $\mathrm{UTF}] \rightarrow$ [Universal Mind with universal consciousness + Matter and Fields \{including thought force $\left.\left(\mathrm{T}_{\mathrm{F}}\right)\right\}+$ TCP \& $\mathrm{TRP}+\mathrm{STC}+\mathrm{UTF}] \rightarrow$ Universal Mind with universal consciousness + Matter and Fields including thought force $\left.\left(\mathrm{T}_{\mathrm{F}}\right)\right\}+$ Life as well as consciousness $+\mathrm{TCP} \& \mathrm{TRP}+\mathrm{STC}$ + UTF [where thought force $\left(\mathrm{T}_{\mathrm{F}}\right)$ is the origin of all the fields, TCP (Though-carrying particle) is the origin of all the field particles, TRP (Thought retaining particle) is the origin of all the matter particles, anti-TRP is the origin of all the anti-particles, STC $=$ Space time continuum and $\mathrm{UTF}=$ Universal thought frequency $\cong$ frequency of the TCP].

Physicists determined that underlying quantum fields give birth to elementary particles. Bhaumik [6] mentioned that Frank Wilczek pointed out, "In quantum field theory, 
the primary elements of reality are not individual particles, but underlying fields. Thus, for example, all electrons are but excitations of an underlying field, naturally called electric field". The same holds true for all the fundamental particles of which matter is made.

The existence of matter depends on the existence of force and vice versa. TCP cannot exist without TRP and vice versa. Many physicists believe that unifying all the forces, including gravity, into a single theory would require a phenomenon called super-symmetry. With supersymmetry, every fermion would have a boson twin, and vice-versa. TCP that behaves like boson should accompany its super-symmetrical partner TRP that functions like fermion in the generalized simpler way. Thus TCP like boson cannot have anti-particle. But TRP that functions like fermion should have its anti-particle and here it is shown as Anti-TRP (see Scheme-I).

\subsection{Pal [7] Explained the Existence of Thought Force and Its Characteristics}

A single field emerged at the origin of the universe containing within itself the blueprint of the physical universe. The primordial single field triggered the onset of the universe. Most physicists believe that a single superforce dominated the first instants of creation. This mysterious super-force is the Thought force $\left(\mathrm{T}_{\mathrm{F}}\right)$ itself. The evolution of all the fundamental fields is originated from this super-force that is the Thought force $\left(\mathrm{T}_{\mathrm{F}}\right)$ in our view.

Physicists determined that underlying quantum fields give birth to elementary particles. Pal [7] explained that thought force $\left(\mathrm{T}_{\mathrm{F}}\right)$ is the primordial quantum field. Thought force $\left(\mathrm{T}_{\mathrm{F}}\right)$ being the primordial quantum field gives birth to TRP that appears to be the origin of all the matter particles. TCP is the carrier of thought force $\left(T_{F}\right)$ that, in turn, appears to be the origin of all the fields. TCP thus appears to be the origin of all the field particles (see Scheme-I). TCP cannot exist without TRP and vice versa. It is to be noted that these TCP and TRP function like wavicle: waveparticle duality.

Thought force $\left(\mathrm{T}_{\mathrm{F}}\right)$, an expression of the universal consciousness, is the primordial quantum field that, in turn, functions as the primary unified field. $T_{F}$ itself may thus be found to be the original single primary unified field that is the origin of all the four fundamental fields along with both the $\mathrm{T}_{\mathrm{F}}$ (micro) and $\mathrm{T}_{\mathrm{F}}$ (macro) as indicated by Pal [7]. This $\mathrm{T}_{\mathrm{F}}$ being an expression of the universal consciousness is applicable to any inanimate object as well as to any biological system (having thinking ability). The quantized energy $\left(\varepsilon_{T}\right)$ of TCP represents universal consciousness. Thus the $\mathrm{T}_{\mathrm{F}}$ being an expression of the quantized energy $\left(\varepsilon_{T}\right)$ of TCP exerts its functions both in vitro and in vivo.

In a purpose to involve both the non-living and living systems of the world, Pal [7] has shown the existences of these TCP, TRP and thought force $\left(\mathrm{T}_{\mathrm{F}}\right)$ in vitro and thought force $\left(\mathrm{T}_{\mathrm{F}}\right)$ in vivo. Anyone can call this TCP by any other name, but as the highly developed living system will have to be evolved in the universe in the long run and as the thought of highly developed living system appears to be a kind of force to be called the thought force $\left(\mathrm{T}_{\mathrm{F}}\right)$ in vivo, $\mathrm{Pal}$ [7] considered it is wise to call it as TCP. Further, as the universe exists along with the universal consciousness that, in turn, is created by the quantized energy $\left(\varepsilon_{T}\right)$ of TCP, we had to use the term TCP.

Pal [7] expressed that the non-living system of the world is governed by the thought force $\left(\mathrm{T}_{\mathrm{F}}\right)$ in vitro and this Thought force $\left(\mathrm{T}_{\mathrm{F}}\right)$ in vitro gives rise to $\mathrm{T}_{\mathrm{F}}$ (micro), $\mathrm{SNF}$, EMF, WNF, GF and $\mathrm{T}_{\mathrm{F}}$ (macro) where $\mathrm{T}_{\mathrm{F}}$ (micro) $=$ Thought force in microcosm, SNF = Strong nuclear force, $\mathrm{EMF}=$ Electromagnetic force, $\mathrm{WNF}=$ Weak nuclear force, $\mathrm{GF}=$ Gravitational force and $\mathrm{T}_{\mathrm{F}}$ (macro) $=$ Thought force in macrocosm. It is to be noted here that $\mathrm{T}_{\mathrm{F}}$ (micro) is a stronger force than the SNF and $\mathrm{T}_{\mathrm{F}}$ (macro) is a weaker force even than the GF.

Pal (7) also expressed that the living system of the world is governed by the thought force $\left(\mathrm{T}_{\mathrm{F}}\right)$ in vivo and this Thought force $\left(\mathrm{T}_{\mathrm{F}}\right)$ in vivo is a type of force that represents the biological 'thought' which is the action of mind. This 'thought' being a type of force controls the 'thought processes' involving the firing of neurons through the quantum mechanical activities of these TCP and TRP in the presence of consciousness. This consciousness, in turn, is the quantized energy $\left(\varepsilon_{T}\right)$ of TCP. The thought force $\left(\mathrm{T}_{\mathrm{F}}\right)$ in vivo is demonstrated in numerous experiments in which thought has an effect on a physical process (often known as mind over matter). This biological 'thought' is a type of force that can cause movement. Controlling movement through thought alone is observed in several experiments conducted by many scientists as indicated by Pal (7). These experiments thus signify the existence of thought force $\left(\mathrm{T}_{\mathrm{F}}\right)$ in vivo.

Pal et al $[3,5]$ showed that the quantized energy $\left(\varepsilon_{T}\right)$ of TCP is responsible to cause the universal consciousness as well as the cosmic microwave background radiation temperature.

\subsection{Existence of Universal Consciousness}

Pal et al [5] and Pal [8] showed the existence of universal consciousness and explained that the quantized energy $\left(\varepsilon_{T}\right)$ of TCP is responsible to cause the universal consciousness as well as the cosmic microwave background radiation temperature. The individual consciousness owes its origin to the universal consciousness created by the same $\varepsilon_{T}$. Many physicists agree with the idea that consciousness is non-local, fundamental in the universe and consciousness is very much a part of the universe, like other objects. It is to be noted that $\mathcal{E}_{T}$, the quantized energy of TCP represents universal consciousness.

Nelson [9], Director of Global Consciousness Project, has observed through the experimentation that coherent consciousness creates order in the world; and subtle interactions link us with each other and the Earth. When human consciousness becomes coherent and synchronized, the behavior of random systems may change. Quantum event based random number generators (RNGs) produce 
completely unpredictable sequences of zeroes and ones. But when a great event synchronizes the feelings of millions of people, our network of RNGs becomes subtly structured. The probability is less than one in a billion that the effect is due to chance. The evidence suggests an emerging noosphere, or the unifying field of consciousness described by sages in all cultures. This evidence signifies and proves the existence of universal consciousness.

The existence of universal consciousness is explained by Radin [10] through experimentation as it is expressed in his book The Conscious Universe: The Scientific Truth of Psychic Phenomena. Further, Consciousness, not matter, is the ground of all existence, declares University of Oregon physicist Goswami through his published (1993) book, "The Self-Aware Universe: How Consciousness Creates the Material World".

As per Penrose [1], consciousness is a part of the universe. Van De Bogart [2] explained, "Since consciousness is a part of the universe it then follows that all consciousness, and the universe, is of the same matrix of energy fields".

\section{TCP and TRP Play the Significant Roles in the So-Called 'Non-Locality' Problem in Quantum Mechanics}

Everything in this universe is interlinked and intertwined through the existence of the quantum mechanical activities of these TCP in the presence of TRP.

The entire Universe or the particles in it are 'connected' through the existence of the quantum mechanical activities of these TCP in the presence of TRP. Pal et al [5] showed that the quantized energy $\left(\varepsilon_{T}\right)$ of TCP is responsible to cause the universal consciousness as well as the cosmic microwave background radiation temperature. The individual consciousness owes its origin to the universal consciousness created by the same $\varepsilon_{T}$.

Pal et al [5] and Pal [8] explained that the universe exists along with the universal consciousness. This universal consciousness exists throughout the universe in the form of universe wide web $(u w w)$ covering fields, particles, spacetime continuum, dark matter, dark energy, void and all its known and unknown parameters along with all its inhabitants (with or without consciousness). The quantized energy $\left(\varepsilon_{T}\right)$ of TCP represents universal consciousness. This universal consciousness is to be taken into account, but usually ignored.

Pal (7) expressed that Thought force $\left(\mathrm{T}_{\mathrm{F}}\right)$ is bridging the microcosm and macrocosm through the generation of a universal web of quantum fields of $T_{F}$ (micro) and $T_{F}$ (macro) due to the existence of the quantum mechanical activities of these TCP in the inherent presence of TRP.

Wilczek [11] expressed that intangible quantum fields fill everything in the universe, including the voids inside atoms and the universe, space between galaxies. Quantum electrodynamics has shown that even the vacuum of space, utterly devoid of matter or conventional forms of energy, actually seethes with activity. The possibility of the existence of a universal web of quantum fields signifies the existence of the $T_{F}$ (micro) and $T_{F}$ (macro) due to the existence of the quantum mechanical activities of these TCP in the inherent presence of TRP.

This universal consciousness functions as a universe wide web (uww) covering the universe as a whole with all its parameters (including void) and inhabitants (with or without consciousness). In this picture, uww of consciousness, quantum concepts like wave particle dualism, position momentum uncertainty, nonlocality and concept of unified field become somewhat understandable as all the entities of this universe are interlinked and intertwined. In this picture, the universal consciousness replaces ether of yesteryears and contains the whole of the universe in its fold.

\section{Discussion}

Pal et al [3, 5] explained, "It is possible that the quantum enigma can be tackled by accepting the existence of the quantum mechanical activities of these TCP and TRP that are the ultimate constituents of any matter as well as any individual mind. The functional state of mind generates consciousness. This consciousness is acting as an interlinking agent between the animate and inanimate". Here thought force $\left(\mathrm{T}_{\mathrm{F}}\right)$ is an expression of the universal consciousness which exists along with the universe. And the quantized energy $\left(\varepsilon_{T}\right)$ of TCP represents universal consciousness.

Bhaumik [6] mentioned, "It would be reasonable to presume that the universe originated in a unity of all fields at or near Planck's dimension. John Wheeler strongly believes that "in defining any useful concept of reality" we have to take into account "the indispensable place of the participating observer---evidenced in quantum mechanics," the foregoing cannot be really true unless consciousness is as essential as aspect of nature as are the fields that give rise to force and matter and the primary field that gave rise to them".

Samanta-Laughton [12] expressed, "At a quantum level particles can exist in many states at a time. What brings them out of this superposition is a matter of debate, but according to the Copenhagen interpretation, it is the act of observing a particle that determines what it is. Bohr went further and said that there is no objective reality 'out there'. Things only exist when we observe them. This implies that the whole universe exists only in our consciousness. Although controversial, the Copenhagen interpretation has stood the test of time with its bizarre philosophical implications".

The reigning tenet of quantum mechanics is the uncertainty principle. A consequence of the uncertainty principle is that the presence of an observer or experimenter determines the outcome of the observation or experiment. Simply stated, this means there is no objective reality; you 'create' what you see through the quantum mechanical activities of these TCP and TRP in the presence 
of consciousness. The quantized energy $\left(\varepsilon_{T}\right)$ of TCP represents universal consciousness. The individual consciousness owes its origin to the universal consciousness created by the same $\varepsilon_{T}$. And these TCP and TRP are the ultimate constituents of any matter as well as any mind in the inherent presence of thought force $\left(\mathrm{T}_{\mathrm{F}}\right)$ in vitro and the thought force $\left(\mathrm{T}_{\mathrm{F}}\right)$ in vivo as it is indicated by Pal et al $[3,5]$.

How could an object's existence depend upon the act of observation? This is due to the fact that an object's existence as well as the act of observation through the prevailing consciousness is totally dependent on the existence of the quantum mechanical activities of these TCP and TRP in the presence of consciousness. The quantized energy $\left(\varepsilon_{T}\right)$ of TCP represents universal consciousness. The individual consciousness owes its origin to the universal consciousness created by the same $\varepsilon_{T}$. And these TCP and TRP are the ultimate constituents of any matter as well as any mind in the inherent presence of thought force $\left(\mathrm{T}_{\mathrm{F}}\right)$ in vitro and the thought force $\left(\mathrm{T}_{\mathrm{F}}\right)$ in vivo as indicated by Pal et al $[3,5]$.

Pal et al $[3,5]$ have expressed mathematically that the cosmic microwave background radiation (CMBR) temperature is due to the quantized energy $\left(\varepsilon_{T}\right)$ of TCP in the inherent presence of TRP. It is expressed that the CMBR temperature exists due to the existence of $\varepsilon_{T}$ of TCP radiated from the radiant mass of universe. The existence of the CMBR temperature confirms the existence of TCP in the inherent presence of TRP. The existence of TCP ensures the existence of thought force $\left(\mathrm{T}_{\mathrm{F}}\right)$. Further, this $\mathrm{T}_{\mathrm{F}}$ exerts its functions both in vitro and in vivo. Ultimately this $\mathcal{E}_{T}$ represents universal consciousness.

$\mathrm{Pal}$ [7] explained the testability for the existence of TCP and TRP along with the Thought force $\left(\mathrm{T}_{\mathrm{F}}\right)$. Pal [7] mentioned several experimental results to signify the functional existence of quantum mechanical activities of these TCP and TRP, the ultimate constituents of matter and mind in the inherent presence of thought force $\left(\mathrm{T}_{\mathrm{F}}\right)$ in vitro and the thought force $\left(\mathrm{T}_{\mathrm{F}}\right)$ in vivo.

\section{Conclusion}

Consciousness in living organisms is a process which involves the quantum mechanical activities of these TCP and TRP, the ultimate constituents of any matter as well as any mind in the inherent presence of thought force $\left(\mathrm{T}_{\mathrm{F}}\right)$ in vitro and the thought force $\left(\mathrm{T}_{\mathrm{F}}\right)$ in vivo as indicated by Pal et al [5].

The 'spooky action-at-a-distance' is built in nature through the existence of the quantum mechanical activities of these TCP in the inherent presence of TRP. The spooky non-local correlations-at-a-distance might evidence a Holy Ghost at work through the existence of the quantum mechanical activities of these TCP and TRP, the ultimate constituents of any matter as well as any mind in the inherent presence of thought force $\left(\mathrm{T}_{\mathrm{F}}\right)$ in vitro and the thought force $\left(\mathrm{T}_{\mathrm{F}}\right)$ in vivo as indicated by Pal et al [5].

\section{References}

[1] R. Penrose, Shadows of the Mind: A Search for the Missing Science of Consciousness. (Oxford University Press) (1994)

[2] W. Van De Bogart, Earth portals: Exploring New Metaphors of Consciousness 1993 willard@earthportals.com

[3] D. Pal and A.U. De, Physics of consciousness and its model may provide guidelines to solve many scientific problems. Neuroquantology 1, 17-28 (2004)

[4] D. Pal, and A.U. De, Consciousness model: Significance of thought-carrying particles and thought-retaining particles in quantum measurement as well as cognitive problem. NeuroQuantology 2, 115-116 (2005)

[5] D. Pal, and A.U. De, The cosmic microwave background radiation temperature signifying the existence of the thought-carrying particle, thought retaining particle and thought force. NeuroQuantology 10: Issue3; 428-442 (September 2012)

[6] M. Bhaumik, Code Name GOD. (Penguin Books India Pvt. Ltd., 11 Community Centre, Panchsheel Park, New Delhi 110017 , India) pp. 132-133; 161-162; 167; 171; 177; 185$186 ; 89-190 ; 198$ (2006)

[7] D. Pal, Existence of thought force and its characteristics. Communicated to American Journal of Modern Physics (AJMP) (2013)

[8] D. Pal, Existence of universal consciousness and its characteristics. Accepted for publication in 2014 International Conference on Advanced Education and Management (ICAEM2014) Beijing, China (2013)

[9] R. Nelson, Director, Global Consciousness Project, Princeton, New Jersey. (2009) rdnelson@princeton.edu

[10] D. Radin, The Conscious Universe: The Scientific Truth of Psychic Phenomena. (Harper Edge) (1997) ISBN 0-06251502-0.

[11] F. Wilczek, Mass Without Mass I: Most of matter. Physics Today 11 (November, 1999)

[12] M. Samanta-Laughton, A Beginner's Guide to Consciousness. The Naked Scientists: Science Radio \& Science Podcasts 2005 